\title{
Recognizing and Preventing Artifacts in Microscopy: A Roundtable Discussion
}

\author{
E. Ann Ellis ${ }^{1}$ and Leona Cohen-Gould ${ }^{2}$ \\ ${ }^{1}$ Consultant in Biological Electron Microscopy, Thomasville, GA \\ ${ }^{2}$ CLC Imaging Core Facility, Weill Cornell Medical College, New York, NY
}

In 1991 the Technologists' Forum presented a symposium on artifacts in biological electron microscopy [1]. Since that time the topic has not been re-visited in our symposia, special topics or rountable discussions. Artifacts are damage caused in specimen preparation and can be confused with specimen ultrastructure. Many artifacts are a result of mechanical or chemical action during sample preparation and some artifacts are due to irradiation by the electron beam during examination of the specimen in the microscope. Recognizing specimen damage is the initial step in preventing the same problem in future preparations and not reporting erroneous data.

Examination of nanoparticles by transmission electron microscopy (TEM) has become an important method for characterizing these particles. Clumping of particles on grids often occurs if grids are not glow discharged before use [to change a hydrophobic surface to a hydrophilic surface] or the solution of the particles is too concentrated. If negative staining is a part of the preparation, there should be attention to the choice of stain, concentration and $\mathrm{pH}$ [2]. Depending on the specimen, drying artifacts may change the characterization of the nanoparticles [3]. Bacterial flagella often drop off if acidic stains are used and some structures can best be preserved by pre-fixation with $1 \%$ (vol/vol) aqueous glutaraldehyde prior to negative staining.

Artifacts in sections can be the result of poor ultramicrotomy skills or problems in embedding. Microtomy problems range from loose fittings with the microtome and specimen, too large block face, improper setup of the cutting arc and dirt or damaged areas on the knife edge. Poor dehydration and infiltration as well as improperly polymerized resins contribute to artifacts in sections [4].

Uranyl acetate is an important reagent in fixation and staining of biological specimens; however, it can also be the source of significant artifacts if used inappropriately. En bloc staining with uranyl acetate extracts glycogen and use of uranyl stains in the presence of phosphate buffers results in needlelike crystals all over the specimen or grid. There are methods to remove these stain artifacts [5, 6]; but it is better not to allow the formation of these artifacts.

Post staining of grids with uranyl acetate followed by lead citrate is a common source of artifacts. One source of problems is old stains which often result in poor staining or require longer staining times which can result in stain precipitates due to drying. The correct $\mathrm{pH}$ of lead stains is 12 and this can best be achieved by using commercially prepared carbonate-free solutions of $1 \mathrm{~N} \mathrm{NaOH} \mathrm{[7].} \mathrm{The} \mathrm{quality} \mathrm{of}$ the water that is used to prepare stains and wash grids is also important. If the laboratory deionized water supply is not of the best quality, it may be necessary to purchase distilled or HPLC grade water just for preparing stains and staining grids.

A perplexing problem with glutaraldehyde and osmium fixation is the presence of electron dense precipitates in sections. The use of divalent cations can result in precipitates of proteins. Magnesium $\left(\mathrm{Mg}^{++}\right)$is preferred to calcium $\left(\mathrm{Ca}^{++}\right)$since it is a smaller molecule and at low concentrations does not precipitate proteins as readily[8]. In some tissues this may be a problem if phosphate buffers are used 
with glutaraldehyde and osmium; however this phenomenon has been observed with cacodylate buffer also. Osmium pepper precipitate can be removed by treating sections with $1 \%$ (wt/vol) periodic acid (freshly prepared) in water for 5-10 minutes followed by several washes in deionized water and then usual post staining [9]. If the formation of the osmium pepper precipitate becomes a recurring problem with the specimen and fixation protocol used, there is a recommendation to eliminate the formation. Inclusion of $0.1 \mathrm{M}$ glycine in buffer washes has been advocated for removing unbound aldehydes from specimens for immunolabel localization and in addition, inclusion of $0.5-1.0 \%$ (vol/vol) dimethyl sulfoxide (DMSO) in buffer washes for cytochemical localizations has been used to rapidly remove unbound fixative. In the buffer washes after aldehyde fixation, add 0-5-1.0\% (vol/vol) DMSO and finally add $0.1 \mathrm{M}(\mathrm{wt} / \mathrm{vol})$ glycine to the final two buffer washes before any other procedures and the final osmication.

Scanning electron microscopy (SEM) has artifacts of charging which indicate that the specimen is not properly grounded and drying artifacts can result from the manner in which specimens are prepared. Critical point drying (CPD) and/or use of chemical critical point drying by use of hexamethyldisilazine (HMDS) eliminates drying artifacts while charging can be reduced by proper coating with heavy metals. Specimens with intricate shapes cannot always be adequately coated with gold or palladium or other heavy metals by sputter coating. Vapor coating with osmium or ruthenium vapors offers a simple way to coat areas of specimens that cannot be reached by sputter coating [10]. In addition, care must be taken in choice of coating material such that there is no interference of peaks in electron spectroscopic analysis.

Over the years we have learned how many artifacts are formed in specimen preparation and how they can be reduced or eliminated. In the event that there are artifacts in specimens, both old and new, that need to be used and cannot be reproduced, there are some salvage methods $[5,6]$ that can be invoked. In addition, once the source of the artifact is identified, protocols should be modified to eliminate the problem.

\section{References:}

[1] R. F. E. Crang and K. L. Klomparens, "Artifacts in biological electron microscopy.”, (Springer, New York) 233 pp.

[2] B. Cao, H. Xu and C. Mao, Microsc. Res. Tech. 74(2011), 627.

[3] B. Michen et al., Scientific Reports 5 (2015), 1.

[4] N. Reid "Ultramicrotomy", ed. AM Glauert (North-Holland Publishing Co., New York) 300 pp.

[5] J. Kuo, G. L. Husca, and LND Lucas, Stain Technol. 56(1981), 221.

[6] E. A. Ellis, in “Electron Microscopy, $3^{\text {rd }}$ Ed." ed. J Kuo (Humana Press, New York) p. 57.

[7] E. S. Reynolds, J. Cell Biol. 17(1963), 208.

[8] A. M. Glauert and P. R. Lewis, "Biological specimen preparation for transmission electron microscopy”, ed. A. M. Glauert (Noth-Holland Publishing Co, New York) 194 pp.

[9] E. A. Ellis and D. W. Anthony, Stain Technol. 54(1979), 282.

[10] P. Echlin 2009. "Handbook of sample preparation for scanning electron microscopy and x-ray microanalysis”, (Springer, New York) 330 pp. 